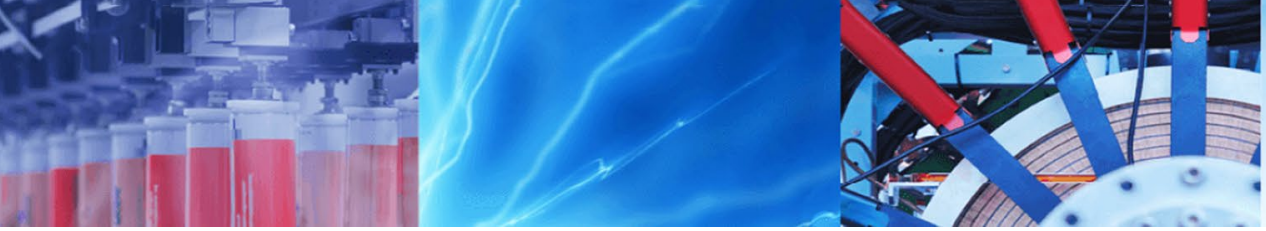

Research Article

\title{
Design and implementation of defected ground surface with modified co-planar waveguide transmission line
}

\author{
Rajshri C. Mahajan ${ }^{1}$ (I) Vini Parashar ${ }^{1} \cdot$ Vibha Vyas $^{1}\left[\right.$ Mukul Sutaone $^{1}$
}

(c) Springer Nature Switzerland AG 2019

\begin{abstract}
This paper proposes a modified Co-Planar Waveguide (CPW) transmission line with Periodic Defected Ground Structures which can be used as a high-speed microwave channel for embedded antennas. CPW ground surface is modified by making slots of various shapes. The modified CPW channels are found suitable for Wi-MAX applications and a particular shape of DGS unit cell can be selected depending on the bandwidth requirement. The CPW transmission line with circular shaped DGS cells of two columns offer a remarkable value of the transmission coefficient of $0.89022(-1.01 \mathrm{~dB})$ therefore it is fabricated and tested. It is observed that both the simulated and tested results agree with each other.
\end{abstract}

Keywords CPW transmission line · Defected ground structures (DGS) - Embedded antenna

\section{Introduction}

Now-a-days, an embedded antenna system is popular due to its work efficiency in rough environment, aesthetic design and less cost. Embedded antennas are either microstrip patch or Printed Circuit Board (PCB) trace antennas. Microwave signals travel through microwave channels or mediums on PCB for efficiently transferring the radiated power from the source to the load with reduced losses. The physical dimensions of PCB trace are prominently related to the wavelength $(\lambda)$ of the microwave signal and most importantly, this PCB trace should have the characteristic impedance value as $50 \Omega$.

These PCB traces are also referred to Co-Planar Waveguide (CPW) transmissions lines for embedded antennas. The transmission lines are used before a Co-Planar Waveguide transmission line such as coaxial transmission line, strip line and microstrip line scale the signal conductor's dimensions to the separation of the ground conductor for setting impedance. Sometimes, this becomes inconvenient when a transmission line has to be connected to different components. This problem is solved by a Co-Planar Waveguide transmission line.

The main characteristic of these CPW transmission lines is to provide a great amount of isolation between the signals, which move through different paths as there is always a Radio Frequency (RF) ground between the traces. Hence, a circuit isolation can be obtained using a CPW transmission line. CPW line supports grounding of shunt elements on the PCB trace which results in a reduced cross-talk with other traces and provides low losses at a very high frequency applications compared to a micro-strip line.

CPW is a transmission line with a ground conductor positioned coplanar to the signal conductor. Therefore, with the help of the ground-gap and line width, its characteristic impedance can be controlled. It implies that the impedance can be kept constant by tapering the width. This is a perfect procedure for matching to a component pin width without changing the substrate thickness.

Co-Planar Waveguide line and Co-Planar Waveguide with a ground surface are the most frequently used CoPlanar Waveguide configurations [1, 2].

Rajshri C. Mahajan, rajshrimahajan2012@gmail.com; Vini Parashar, vinip16.extc@coep.ac.in; Vibha Vyas, vsv.extc@coep.ac.in; Mukul Sutaone, mssutaone.extc@coep.ac.in| ${ }^{1}$ Department of Electronics and Telecommunication, College of Engineering Pune (COEP), Pune, Maharashtra 411005, India. 
In the high-speed microwave circuits, it is required to transmit various frequency signals from one point of $P C B$ to another without any interference in the performance of the overall circuit and signals. This requirement of high speed microwave circuits can be realized using CPW line with slight modifications to obtain a frequency dependent behaviour [3]. The modifications are based on Defected Ground Structures (DGS) which are realized by 2-Dimensional etched symmetric areas on the metallic ground surface or on the signals trace of a CPW line. The transmission line with DGS has a unique property to prevent the propagation of electromagnetic waves in a specific frequency band along the transmission line [4].

There are two major classes of DGS viz. DGS with a single defect and DGS with a small number of defects in a periodic or non-periodic manner. In the recent past, different shapes of DGS were used for the modification of a CPW line but are mostly based on a single defect. CPW line with two slots along the line that generates a Transmission Zero (TZ) in the transmission response is printed on a fixed substrate. A metallic patch is also printed on the bottom face of a movable substrate located on the fixed substrate $[5,6]$.

In literature, a reconfigurable DGS resonator fabricated on CPW technology was presented too. The chosen design was indeed based on a slot defect created on the lateral ground planes of CPW [7].

In another reference, a CPW fed microstrip antenna with a defected crown shaped substrate was designed. The radiating patch of the proposed antenna was taken in the form of an extended U-shape [8]. A spiral-shaped DGS for CPW (DGSCPW), which was used as a kind of periodic structure for a planar transmission line, was proposed. The proposed spiral-DGSCPW adopts spiral-shaped defects on both ground planes of CPW [9].

The implementation of a lattice shape of the CPW DGS unit cell which was located on the lateral metallic ground plane was carried out. CPW band-pass filter was modified such that it exhibited as band-gap characteristics using one or two series connection of DGS to improve its bandwidth [10].
All these studies were mainly based on a single defect on a ground plane. There was also a proposed two- dimensional (2D) PDGS for CPW (PDGSCPW) based on the repetition of a unit-cell in a simplified and systematic way [11]. For this periodic DGSCPW structure, the standard dumbbell structure was used [12].

In the recent study related to DGS, the structure was modified and used for 5G MIMO antenna and imaging applications for communication and medical fields [13-15].

In the proposed research work, a novel CPW transmission line is presented based on a small number of defects in a periodic manner. To improve the parameters of CPW line, various shapes of unit cells were designed and their effects were studied depending on the number of columns of DGS cells along the transmission line.

The main contributions of this research study are:

- Implementation of a transmission line with a Periodic Defected Ground Surface using periodic defects of shapes like $C$, square, circle and hexagon,

- Parametric study of the number of columns of each shaped defect,

- Fabrication of a transmission line with circular shaped defects on a ground surface to validate the simulation results

The organisation of the paper is as follows: Sect. 2 elaborates on the introduction of a CPW transmission line and DGS. Section 3 presents the experimentations and results. Section 4 covers validation with a fabricated CPW transmission line. Section 5 offers conclusions followed by the literature referred in form of 'References'.

\section{CPW transmission line structure and DGS}

\subsection{CPW transmission line structure}

Figure 1 a shows a substrate loaded CPW transmission line.

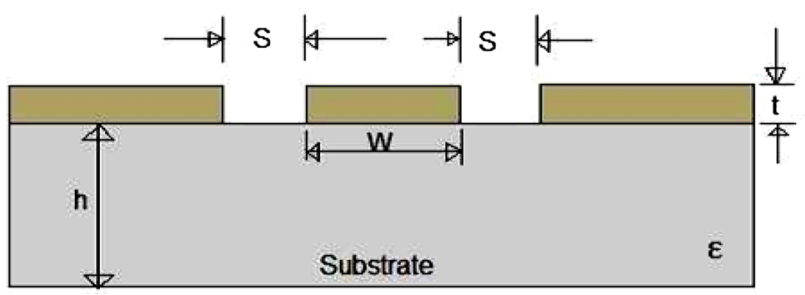

(a)

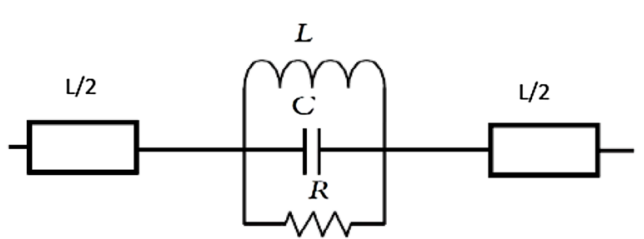

(b)

Fig. 1 a Co-Planar Waveguide transmission line, $\mathbf{b}$ Equivalent circuit of DGS 
Considering Fig. 1 a of CPW line, ' $w$ ' is the width of track, ' $s$ ' is the width of the gap between line and ground surface, ' $h$ ' is the thickness of dielectric, ' $\varepsilon$ ' is permittivity of substrate, ' $t$ ' is the thickness of a metallic patch or PCB trace.

The size reduction of the CPW line is possible without limit at the cost of higher losses. The ratio of a centre strip width ' $w$ ' to the gap width 's' decides the characteristic impedance of this transmission line; this is the main reason of an unlimited size reduction. The infinite range of ' $w$ ' and 's' values is available which makes the CPW line design unique and this results into a specific impedance requirement. This can be advantageous, especially when designing the CPW line at high frequencies.

The characteristic impedance, $Z_{0}$ of the CPW transmission line is calculated using Eq. (1)

$Z_{0}=\frac{60.0 \pi}{\sqrt{\varepsilon_{e f f}}\left\{\frac{K(k)}{K\left(k^{\prime}\right)}+\frac{K(k l)}{K\left(k^{\prime}\right)}\right\}}$

where $\mathrm{a}=$ Track width (s)

$b=w+2 s$

$k=\frac{a}{b}$

$k^{\prime}=\sqrt{1-k^{2}}$

$k l^{\prime}=\sqrt{1-k l^{2}}$

$k l=\frac{\tanh \frac{\pi a}{4 h}}{\tanh \frac{\pi b}{4 h}}$

$\varepsilon_{\text {eff }}=\frac{1+\varepsilon_{r} \frac{K\left(k^{\prime}\right)}{K(k)} \frac{K(k l)}{K\left(k l^{\prime}\right)}}{1+\frac{K\left(k^{\prime}\right)}{K(k)} \frac{K(k l)}{K\left(k l^{\prime}\right)}}$

In Eqs. (1 to 7), the complete elliptic integral is defined by $\mathrm{K}$. The ratio of the complete elliptic functions in equation may be approximated by Eq. 8 .

$\frac{K(k)}{K\left(k^{\prime}\right)}=\left\{\begin{array}{c}\frac{1}{2 \pi} \ln \left[2 \frac{\sqrt{1+k}+\sqrt[4]{4 k}}{\sqrt{1+k}-\sqrt[4]{4 k}}\right] \quad \text { for } 1 \leq \frac{k}{k^{\prime}} \leq \infty, \frac{1}{\sqrt{2}} \leq k \leq 1 \\ \frac{2 \pi}{\ln \left[2 \frac{\sqrt{1+k^{\prime}}+\sqrt[4]{4 k^{\prime}}}{\sqrt{1+k^{\prime}}-\sqrt[4]{4 k^{\prime}}}\right]} \quad \text { for } 0 \leq \frac{k}{k^{\prime}} \leq 1,0 \leq k \leq \frac{1}{\sqrt{2}}\end{array}\right.$

The above mathematical equations of a CPW line helps in understanding the characteristic features of the designed transmission line [16].

\subsection{Defected ground surface (DGS)}

DGS is an etched periodic or non-periodic ground surface of a transmission line which reduces the size of microwave components. This methodology is used for improving the properties of antenna parameters like frequency, bandwidth, etc. Periodic DGS with microstrip lines has bandpass and low-pass planar filters. The antenna achieves a greater degree of miniaturization using a periodic defect. The periodic structure is referred to as the repetition of a single defect with finite spacing. The cascading of resonant cells in a ground surface results into higher bandwidth along with an improved return loss. Figure $1 \mathrm{~b}$ shows an equivalent circuit of DGS [17-19].

DGS acts as a parallel RLC circuit. The defects which are comparable to the wavelenth cause the current paths to elongate and therefore behave like an inductor. The potential is stored in the defects making it a capactor while the losses in the defect act as a resistor. Figure $1 \mathrm{~b}$ shows the equivalent circuit of the unit cell DGS.

DGS is implemented majorly using a single defect or a small number of defects in a perioidic or non-periodic manner. In the recent past, different shapes of DGS were used for the modification of a CPW line but they are mostly based on a single defect [20].

The CPW line in presence of DGS offers many advantages over microstrip lines. This combination provides the facility to have the signal and ground on the same surface and can easily be integrated with RF circuits without the need of via holes. Additionally, the combination is insensitive to substrate thickness and substrate dielectric constant $[3,20]$.

\section{Experimentations, results and analysis}

This section presents experimentations and performances of CPW transmission line with variations in shapes of DGS cells. The reflection and transmission coefficients or return and transmission loss $\left(\mathrm{S}_{11}\right.$ and $\left.\mathrm{S}_{21}\right)$ are used to elaborate the characteristics of the respective CPW transmission line and its modifications are carried out using PDGS. These experiments are optimized for the lowest value of reflection coefficient and the highest value of transmission coefficient, in other words, the lowest return loss $(\mathrm{dB})$ and the highest transmission gain $(\mathrm{dB})$.

\subsection{Design of simple CPW transmission line}

A $50 \Omega$ simple CPW transmission line with a design specification of $100 \mathrm{~mm} \times 50 \mathrm{~mm} \times 1.6 \mathrm{~mm}$ is designed. The substrate is FR-4 with a relative permittivity of 4.4. The design is simulated using Ansoft's High Frequency Structure 
Simulator Version 11 (HFSS 11) and is configured for the entire operating frequency range from 1 to $10 \mathrm{GHz}$. The transmission line is applied with two wave ports in HFSS for obtaining $\mathrm{S}_{11}$ and $\mathrm{S}_{21}$ parameters. The radiation air box is applied around the entire simulated design.

Figure 2 a shows a slight tilted top view showing the ports present on either side of the CPW line while Fig. 2 $b$ shows the dimensions of the standard CPW line where ' $w$ ' is the width of the CPW line and ' $g$ ' is the gap present between the CPW line and the coplanar ground plane.

The width ( $w$ ) of the strip and the gap $(\mathrm{g})$ between the strip and the coplanar ground plane of the CPW line are optimized to be $3 \mathrm{~mm}$ and $0.2 \mathrm{~mm}$ respectively to obtain $50 \Omega$ impedance using Eqs. (1) to (7). The simulated reflection coefficient $\left(S_{11}\right)$ graph gives $S_{11}$ value of $-44.75 \mathrm{~dB}$ at $7.18 \mathrm{GHz}$ frequency. The return loss/impedance matching is fulfilled from 6.891 to $7.406 \mathrm{GHz}$ i.e., for $513 \mathrm{MHZ}$ with the help of a CPW microstrip line.

To enhance the impedance bandwidth, reflection and transmission coefficient values, modifications are proposed in the standard CPW line. In this paper, various shapes of slots are etched on a coplanar ground plane on either side which acts as DGS.

\subsection{Design of CPW line with C-shaped DGS}

The CPW line with physical dimensions as explained in Sect. 3.1, are considered and C-shaped cells are etched as shown in Fig. 3 a. The $C$ shaped DGS unit cell is designed by creating three rectangles and then uniting them. There are 24 numbers of $C$-shaped cells which are arranged in a periodic manner in such a way that the geometry along the axes of the CPW line is symmetrical. The dimensions of unit C-shaped cell are shown in Fig. $3 \mathrm{~b}$ where $C_{l}$ is the length of the shape, $C_{b}$ is the breadth of $C$ leg, $C_{g}$ is the gap between the two $C$ shapes along the line and $d$ is the distance between C-shape and the gap (g). Each DGS cell is surrounded by a small air box to avoid a false resonance at a certain frequency. As a C-shape is formed from the combination of three rectangles, the dimensions are referred to the length and the breadth of the rectangle. Table 1 shows the dimensions of the $\mathrm{C}$-shaped unit cell.

Figure $3 \mathrm{~b}$ shows the dimensions of the unit $\mathrm{C}$-shaped cell and relative measurements.

Figure $3 \mathrm{c}$ shows the return and transmission loss for single-column C-shaped cells. From the graph, it is found that there are three resonating bands at 7.22, 7.94 and $8.43 \mathrm{GHz}$ with transmission bandwidth of $1.78 \mathrm{GHz}$. To study the effect of multiple numbers of columns of the C-shaped cell, the same design is simulated with two and three columns of the $C$ - shaped cells.

Figures 4 a, b show CPW line with two and three columns of $C$-shaped DGS respectively. Figure $4 \mathrm{c}$ shows the distance $G_{c}$ between the two C-shaped cells which is $1 \mathrm{~mm}$.

Figure 5 a shows the graph of reflection coefficient and transmission coefficient according to which the return loss for two columns of the C-shaped DGS is found to be $-34.80 \mathrm{~dB}$ at $8.14 \mathrm{GHz}$ and the transmission gain of $-3.34 \mathrm{~dB}$ is obtained. By comparing it with single column results, it is seen that the return loss is improved and it is found below $-10 \mathrm{~dB}$ from 6.91 to $8.48 \mathrm{GHz}$ i.e., the impedance bandwidth is $1.57 \mathrm{GHz}$.

From Fig. 5 b, it can be noticed that the return loss obtained is $-24.47 \mathrm{~dB}$ resonating at $7.51 \mathrm{GHz}$ and transmission gain is $-3.73 \mathrm{~dB}$. The transmission gain obtained is better for three columns of C-shaped DGS as compared to two and single column C-shapes DGS designs. Additionally, the return loss found is below $-10 \mathrm{~dB}$ from 6.84 to $8.30 \mathrm{GHz}$ i.e., the bandwidth calculated is $1.46 \mathrm{GHz}$. The obtained results are better for three column C-shaped DGS in terms of bandwidth and transmission coefficient which are tabulated in Table 2.

\subsection{Design of CPW line with square shaped DGS}

This section proposes a square shaped DGS. The cells are in a periodic manner and are added in such a way that the geometry along the axes of the square shape is kept constant. The dimensions of the unit square cell are optimized to obtain a transmission impedance of $50 \Omega$ which is shown in Table 3.
Fig. 2 Simple CPW line a Tilted view of the CPW line $\mathbf{b}$ Dimensions of the CPW line

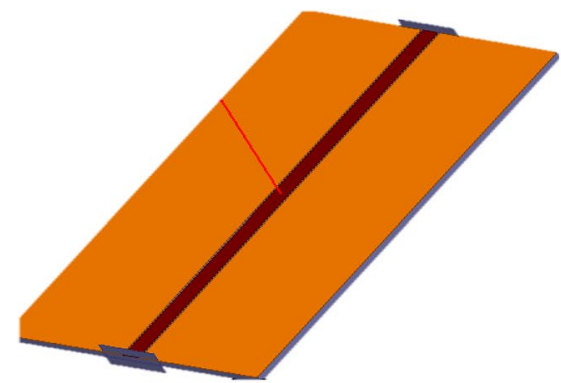

(a)

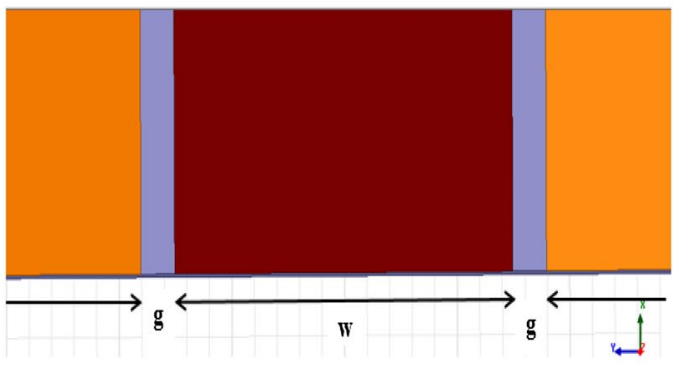

(b) 
Fig. 3 a CPW line with a periodic $C$ shaped DGS on either side of the line $\mathbf{b}$ Dimensions of $C$ shaped DGS cell c $\boldsymbol{S}_{11}$ and $\boldsymbol{S}_{21}$ graph

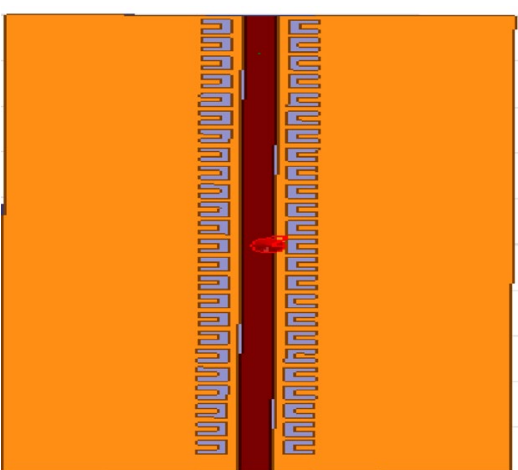

(a)

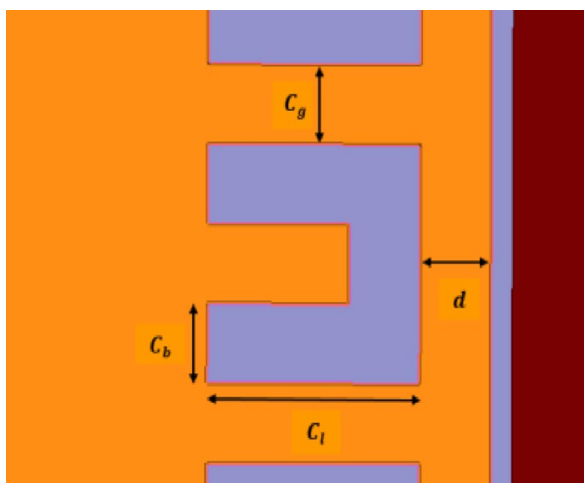

(b)

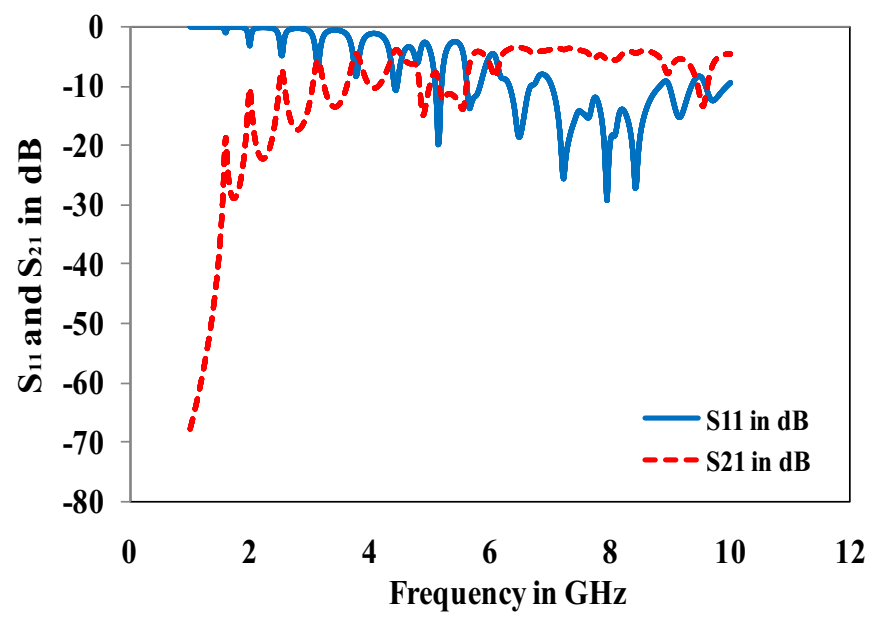

(c)

Table 1 Dimensions of the C-shaped DGS

\begin{tabular}{ll}
\hline Parameter & $\begin{array}{l}\text { Dimen- } \\
\text { sion } \\
(\mathrm{mm})\end{array}$ \\
\hline length of the shape $\left(C_{l}\right)$ & 3 \\
breadth of $C$ leg $\left(C_{b}\right)$ & 1 \\
gap between the two $C$ shapes $\left(C_{g}\right)$ & 1 \\
distance between the $C$ shape and the gap $(d)$ & 1 \\
\hline
\end{tabular}

Figure 6 a shows CPW line with a square shaped periodic DGS on either side of the line. In this case too, the results are analyzed and compared by increasing the number of columns of periodic square shaped DGS. Figure $6 b$ shows the dimensions of a square shape DGS cell where $S_{\text {, }}$ is the length or the side of a square shaped cell, $S_{g}$ is the gap between the two square cell along the column and $d$ is the distance between the square shaped DGS column and the gap (g).

Table 3 shows the optimized dimensions chosen for simulation. Figure $6 \mathrm{c}$ shows a reflection coefficient graph which gives a return loss of $-31.52 \mathrm{~dB}$ at a resonant frequency of $7.81 \mathrm{GHz}$ and the transmission gain is $-4.428 \mathrm{~dB}$. By the same graph bandwidth of the square shaped DGS, a single column is calculated to be $1.35 \mathrm{GHz}$ using the graph.

In this case, it is seen that only the transmission coefficient is found to be very less. For a comparison purpose and to enhance the transmission coefficient, the number of columns is increased to two and three. Figures $7 \mathrm{a}, \mathrm{b}$ show the CPW line with two and three columns of a square shaped DGS. Figure $7 \mathrm{c}$ shows the distance $G_{s}$ between the two square shaped cells.

Figure 8 a shows the graph of a reflection coefficient and transmission coefficient according to which return loss for two columns square shaped DGS is found to be $-21.91 \mathrm{~dB}$ at $8.86 \mathrm{GHz}$ and transmission gain is $-3.889 \mathrm{~dB}$. It is seen that the gain increases compared to the single column of square shaped DGS. By analyzing the return loss graph, the impedance bandwidth calculated is $1.93 \mathrm{GHz}$ which is the greatest bandwidth found till now for different simulations. In Fig. 8 b, the return loss obtained is $-26.26 \mathrm{~dB}$ resonating at $8.31 \mathrm{GHz}$ and the transmission gain obtained is $-3.188 \mathrm{~dB}$. The transmission coefficient obtained is better compared to the two and single-column 


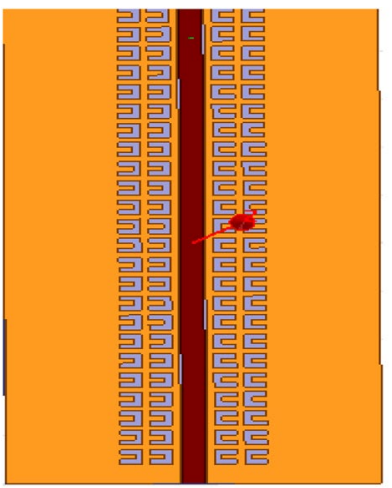

(a)

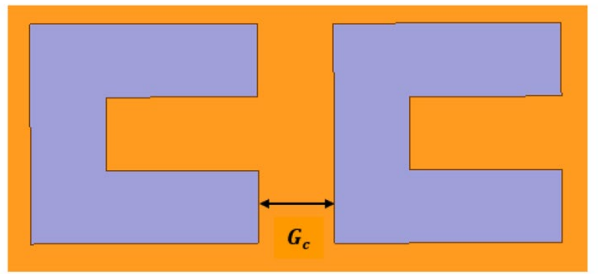

(c)

Fig. 4 a CPW line with two column C-shaped DGS b CPW line with three column $\mathrm{C}$-shaped DGS $\mathbf{c}$ dimension between two $\mathrm{C}$-shapes along the column

square-shaped DGS designs. Further, the return loss found is below $-10 \mathrm{~dB}$ from 6.87 to $8.40 \mathrm{GHz}$ i.e., the bandwidth calculated is $1.53 \mathrm{GHz}$. By comparing the three designs, it can be said that the two and three column DGS are better than a single column square DGS. If the transmission gain is considered, then three column DGS is better and if the bandwidth is considered then two-column DGS is better (Table 4).

\subsection{Design of CPW line with circle shaped DGS}

The circular shaped DGS is proposed with the same number of columns as explained in Sects. 3.2 and 3.3. There are 25 cells of circular shaped slots on both the sides of the
Table 2 Comparison of C-shaped DGS based on the number of columns

\begin{tabular}{lllll}
\hline Design & $\boldsymbol{S}_{11}(\mathrm{~dB})$ & $\boldsymbol{f}_{\boldsymbol{r}}(\mathrm{GHz})$ & $\boldsymbol{S}_{21}(\mathrm{~dB})$ & $\begin{array}{l}\text { Impedance } \\
\text { bandwidth }\end{array}$ \\
\hline $\begin{array}{l}\text { Single column C-shaped } \\
\text { DGS }\end{array}$ & -25.51 & 7.22 & -3.87 & $1.78 \mathrm{GHz}$ \\
$\begin{array}{l}\text { Two column C-shaped } \\
\quad \text { DGS }\end{array}$ & -34.80 & 8.14 & -4.15 & $1.57 \mathrm{GHz}$ \\
$\begin{array}{l}\text { Three column C-shaped } \\
\text { DGS }\end{array}$ & -24.47 & 7.51 & -3.73 & $1.46 \mathrm{GHz}$ \\
\hline
\end{tabular}

Table 3 Dimensions of square shaped DGS

\begin{tabular}{ll}
\hline Parameters & Units $(\mathrm{mm})$ \\
\hline$S_{I}$ & 1.2 \\
$S_{g}$ & 0.8 \\
$d$ & 1 \\
\hline
\end{tabular}

CPW line. Figure 9 a shows the CPW line with a periodic circle shaped DGS. Initially, the single column of these circle shaped periodic DGS is analysed then for the purpose of comparison, these columns are increased. Figure $9 \mathrm{~b}$ shows the dimensions of the circle shaped where $C_{i r}$ is the radius of the circle, $C_{i g}$ is the gap between the two circle shaped DGS along the line and $d$ is the distance between the circle shape and the gap (g). Table 5 shows the dimensions of a circle shaped DGS cell.

Figure $9 \mathrm{c}$ shows the return loss graph, which is found to be $-30.13 \mathrm{~dB}$ at $8.34 \mathrm{GHz}$ frequency. Return loss is below $10 \mathrm{~dB}$ from 7.90 to $8.62 \mathrm{GHz}$ i.e. bandwidth results as $0.72 \mathrm{GHz}$ and the transmission gain is $-5.28 \mathrm{~dB}$.

The above-mentioned design is for a single column of circle shapes along the line, for the purpose of comparison, the number of columns is increased. The simulations are performed for two and three columns of a circle shaped DGS along the line. Figures $10 a$, $b$ show the CPW line with two and three columns of a circle shaped DGS. Figure 10 c shows the distance $G_{c i}$ between the two circles shaped cell which is $0.6 \mathrm{~mm}$.
Fig. 5 a $\boldsymbol{S}_{11}$ and $\boldsymbol{S}_{21}$ graph of CPW line with two columns of C-shaped DGS b $\boldsymbol{S}_{11}$ and $\boldsymbol{S}_{21}$ graph of three columns of Cshaped DGS

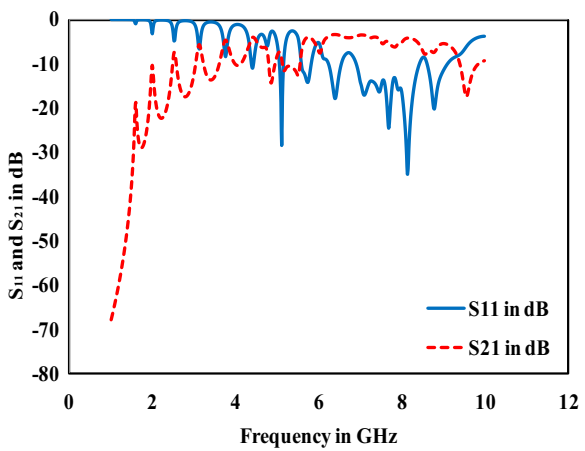

(a)

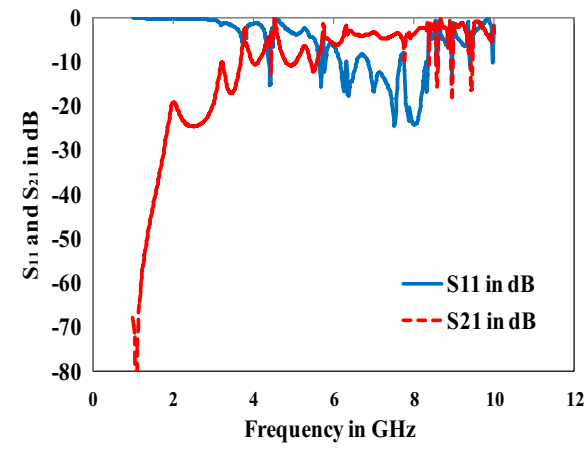

(b) 
Fig. 6 a CPW line with square shaped DGS on either side $\mathbf{b}$ Dimensions of square shaped DGS cell $\boldsymbol{c} \boldsymbol{S}_{11}$ and $\boldsymbol{S}_{21}$ graph of single column square shaped DGS

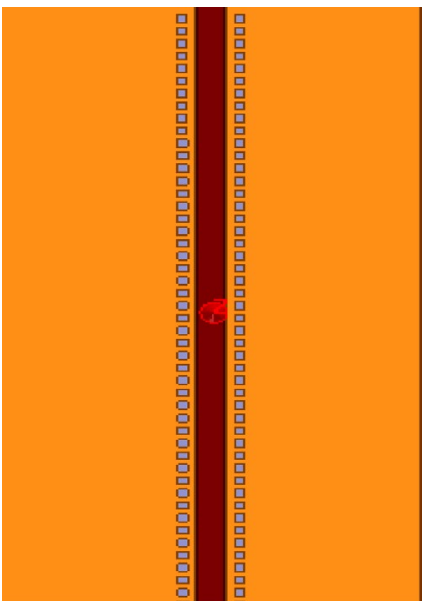

(a)

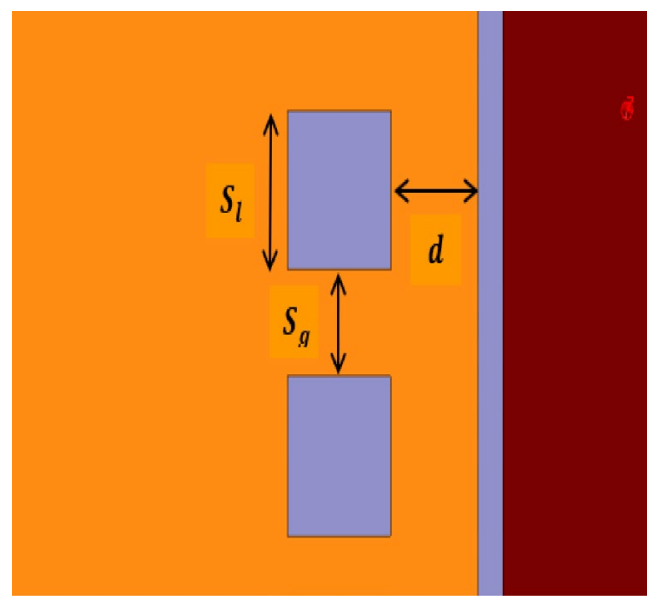

(b)

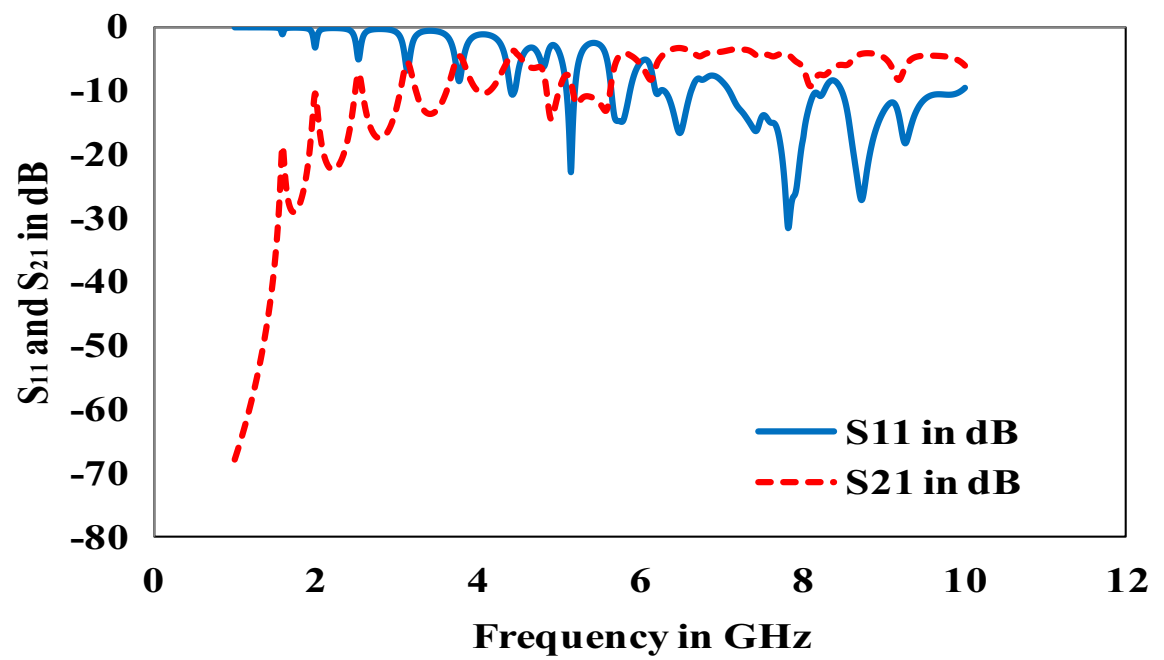

(c)

Figure 11a shows a graph of a reflection coefficient and transmission coefficient according to which the return loss for two column circle shaped DGS is found to be $-30.69 \mathrm{~dB}$ at $8.53 \mathrm{GHz}$ and the gain of $-1.01 \mathrm{~dB}$ is obtained. By comparing it with a single column, it is seen that both the return loss and transmission loss both are improved and return loss is found below $-10 \mathrm{~dB}$ from 6.91 to $8.48 \mathrm{GHz}$ i.e., the bandwidth is $1.77 \mathrm{GHz}$.

Figure $11 \mathrm{~b}$ shows a graph of a reflection coefficient and transmission coefficient according to which the return loss for the two column circle shaped DGS is found to be $-20.57 \mathrm{~dB}$ at $7.43 \mathrm{GHz}$ and the gain of $-4.91 \mathrm{~dB}$ is obtained. By comparing it with a single column, it is seen that both the return and transmission loss are improved and the return loss is found below $-10 \mathrm{~dB}$ from 6.91 to $8.48 \mathrm{GHz}$ i.e., the bandwidth is $1.97 \mathrm{GHz}$ (Table 6).

\subsection{Design of CPW line with hexagonal shaped DGS}

In simulations for hexagonal shaped DGS, the specifications are same like the simple CPW line. The same kind of modifications is performed for hexagonal shape as suggested for the previous shapes. The hexagonal shaped DGS cells are used here on either side of $50 \Omega$ transmission line. The cells are in a periodic manner and are added in such a way that the geometry along the axes of the hexagonal shape is kept symmetrical. The number of hexagonal shaped DGS used on either side of the transmission line is twelve.

Figure 12 a shows a CPW line with a hexagonal shaped periodic DGS on either side of the line. In this case too, the results are analysed and compared by increasing the number of columns of a periodic square shaped DGS. Figure 12 


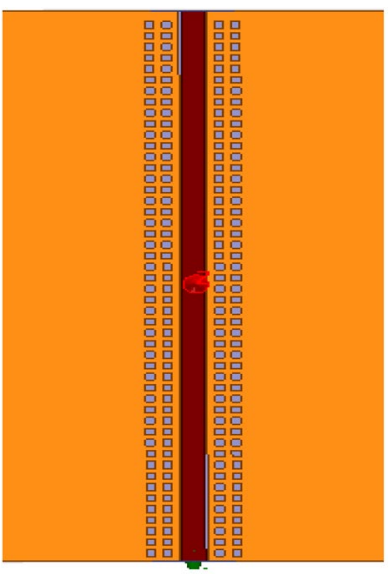

(a)

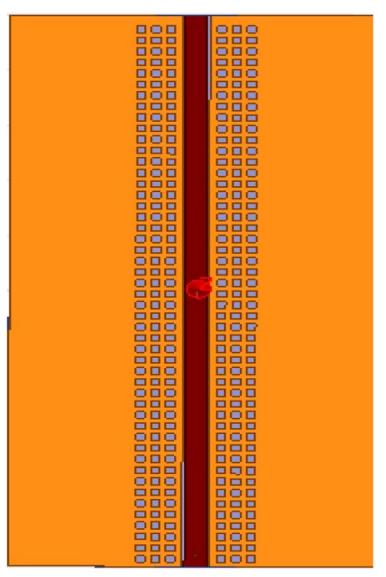

(b)

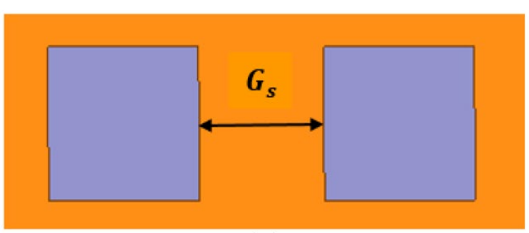

(c)

Fig. 7 a CPW line with two column of square shaped DGS b CPW line with three column of square shaped DGS c Horizontal distance between the two columns

b shows the dimensions of the hexagonal shaped DGS cell where $H_{l}$ is the length of the hexagon or a vertical dimension of the hexagon shaped cell, $H_{b}$ is the breadth or horizontal dimension of hexagon, $H_{g}$ is the gap between the two-hexagon cell along the column and $d$ is the distance between the hexagonal shaped DGS column and the gap (g).
Table 7 shows the optimized dimensions which are chosen for simulation. Figure $12 \mathrm{c}$ shows the reflection coefficient graph which gives a return loss of $-27.88 \mathrm{~dB}$ at a resonant frequency of $6.48 \mathrm{GHz}$ and the transmission loss is $-3.37 \mathrm{~dB}$. By the same graph, bandwidth of the hexagon shaped DGS with a single column is calculated.

In this case, it is seen that the transmission coefficient is not up to the mark and the bandwidth obtained is small. For the purpose of comparison, again and to enhance the transmission gain and the bandwidth, the number of columns is increased to two and three respectively.

Figures $13 \mathrm{a}$, b show the CPW line with two and three columns of hexagonal shaped DGS. Figure $13 \mathrm{c}$ shows the distance $G_{h}$ between the two-hexagonal shaped cells which is $0.6 \mathrm{~mm}$.

Figure 14 a shows a graph of reflection coefficient and transmission coefficient according to which the return loss for the two columns of hexagonal shaped DGS is found to be $-23.96 \mathrm{~dB}$ at $8.71 \mathrm{GHz}$ and the gain received is $-5.689 \mathrm{~dB}$. After analysing, the return loss graph, it is found below $10 \mathrm{~dB}$ from 6.87 to $8.95 \mathrm{GHz}$ i.e., the bandwidth calculated is $2.08 \mathrm{GHz}$.

From Fig. 14 b, the return loss obtained is $-23.11 \mathrm{~dB}$ resonating at $6.80 \mathrm{GHz}$ and the transmission loss obtained is $-3.35 \mathrm{~dB}$. The transmission loss obtained is better compared to the two and single column hexagonal shaped DGS designs. The return loss found is below $-10 \mathrm{~dB}$ from 6.23 to $8.26 \mathrm{GHz}$ i.e., the bandwidth calculated is $2.03 \mathrm{GHz}$. By comparing the three designs, it can be said that the two and three-column DGS are better than a single-column hexagonal shaped DGS. If the gain is considered, then a three column DGS is better and if bandwidth is considered, then a two column DGS is better (Table 8).
Table 4 Comparison of square shaped DGS based on the number of columns

\begin{tabular}{lllll}
\hline Design & $\boldsymbol{S}_{11}(\mathrm{~dB})$ & $\boldsymbol{f}_{\boldsymbol{r}}(\mathrm{GHz})$ & $\boldsymbol{S}_{21}(\mathrm{~dB})$ & $\begin{array}{c}\text { Impedance } \\
\text { bandwidth }\end{array}$ \\
\hline Single column square shaped DGS & -31.52 & 7.81 & -.428 & $1.35 \mathrm{GHz}$ \\
Two column square shaped DGS & -21.91 & 8.86 & -3.889 & $1.93 \mathrm{GHz}$ \\
Three column square shaped DGS & -26.26 & 8.31 & -3.188 & $1.43 \mathrm{GHz}$ \\
\hline
\end{tabular}

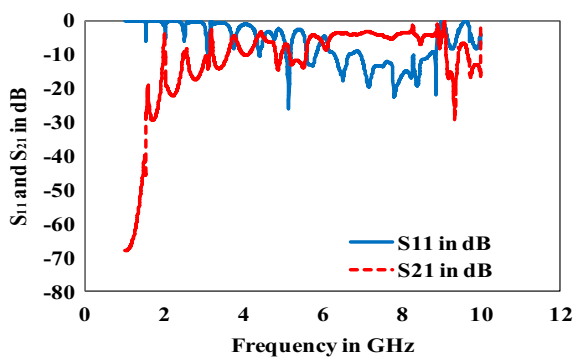

(a)

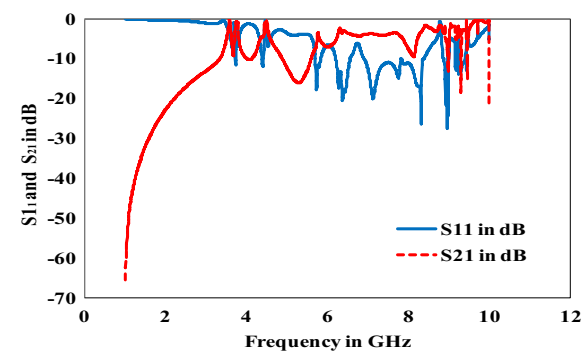

(b) 


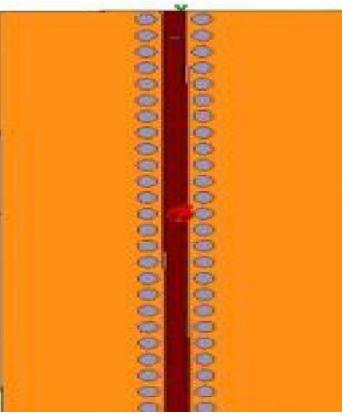

(a)

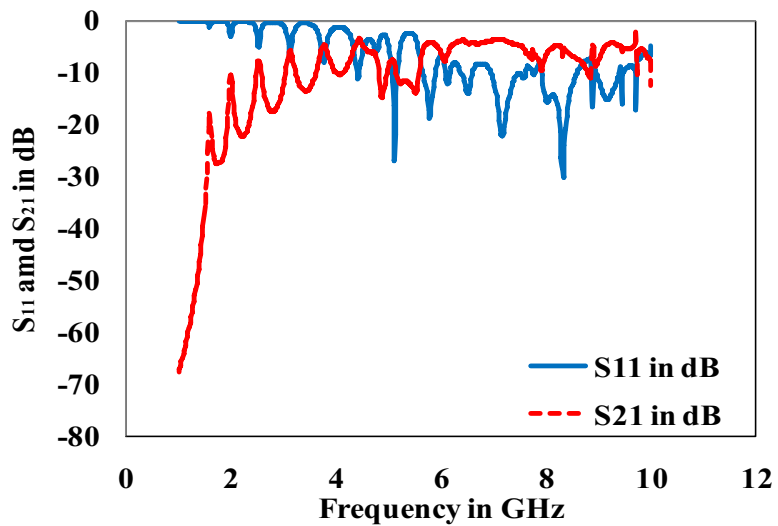

(c)

Fig. 9 a CPW line with a circle shaped DGS on either side b Dimensions of a circle shaped DGS cell $\mathbf{c} \boldsymbol{S}_{11}$ and $\boldsymbol{S}_{21}$ graph of single column circle shaped DGS

Table 5 Dimensions of circle shaped DGS

\begin{tabular}{ll}
\hline Parameter & Units $(\mathrm{mm})$ \\
\hline$C_{\text {ir }}$ & 1.2 \\
$C_{i g}$ & 1.6 \\
$d$ & 1 \\
\hline
\end{tabular}

\section{Fabrications and validation}

In Sect. 3, the CPW line with a periodic DGS is analysed and its performance is tested for various shapes and the number of columns of DGS cells. The $S_{11}$ (return loss) and $S_{21}$ (transmission gain) are obtained as the simulated results of each experiment. From the observations, it is found that a circular-shaped DGS with two columns gives both these values at an optimum level. The circular shape is a naturally occurring shape and it has a uniform DGS coupling. As the sharp edges are absent on a circle shape, there is a reduced diffraction loss. Therefore, the transmission gain is higher compared to other shapes. Secondly, the curvature of a circular shaped DGS exposed to the transmission is larger compared to

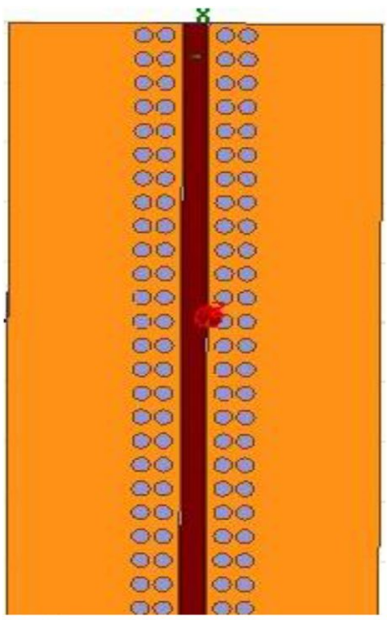

(a)

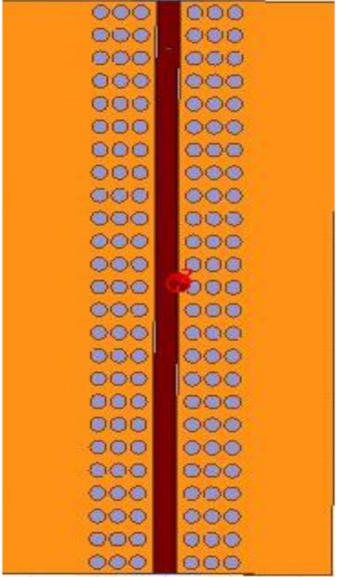

(b)

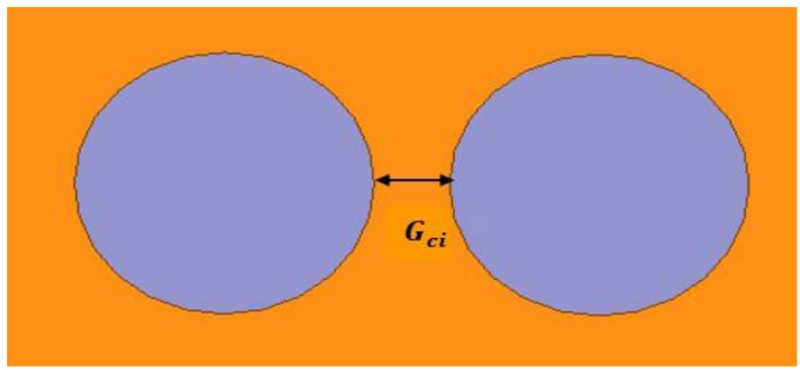

(c)

Fig. 10 a CPW line with two columns of a circle shaped DGS b CPW line with three columns of circle shaped DGS $\mathbf{c}$ Horizontal distance between the two columns

other shapes. This results into a considerable reduction in fringing fields which improves the transmission gain. Hence, this design is fabricated for the purpose of testing and validation using the dimensions explained in 3.4. Figure 15 a shows a photograph of fabricated CPW line with two columns of the circle shaped DGS.

The fabricated CPW transmission line is tested using $\mathrm{R}$ \& S ZNB Vector Network Analyzer (VNA) for validating $S_{11}$ graph. The losses because of the material used for CPW line fabrication and soldering of the connector are considered for the calibration of the connecting cables.

Figure $15 \mathrm{~b}$ shows a plot of a return loss measured result using VNA and the simulated result. Table 9 shows a comparison of both the measured and simulated results and it is found that the return loss found in the measured result is $-30.06 \mathrm{~dB}$ whereas in the simulation result, it is $-31.18 \mathrm{~dB}$. The resonant frequency for the measured result is obtained as $7.81 \mathrm{GHz}$ and for the simulated result it is $7.65 \mathrm{GHz}$. The bandwidth obtained from the simulated result is $1.43 \mathrm{GHz}$ $(6.87-8.30 \mathrm{GHz})$ whereas in the measured result it is 1.10 $(+) \mathrm{GHz}$. Here, (+) means that it is greater than the tested 
Fig. 11 a $S_{11}$ and $S_{21}$ graph of CPW line with two columns of circle shaped DGS b $\boldsymbol{S}_{11}$ and $\boldsymbol{S}_{21}$ graph of three columns of circle shaped DGS

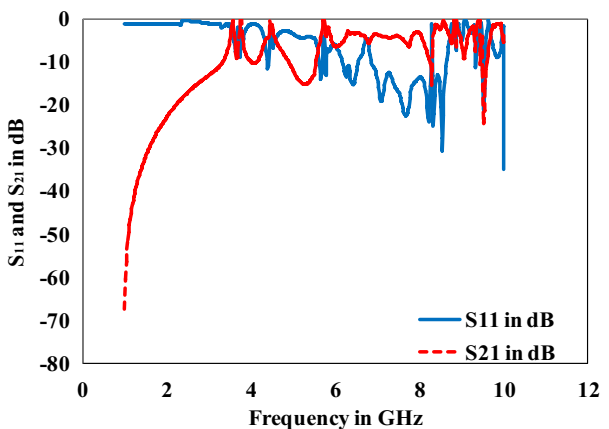

(a)

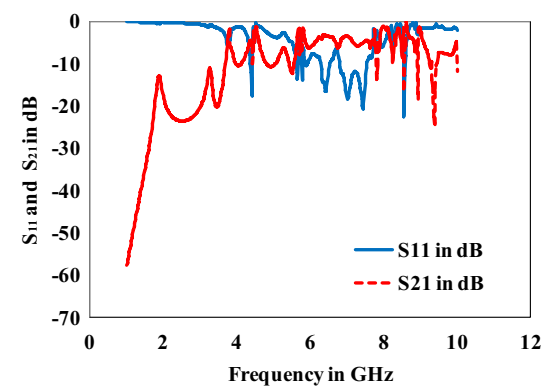

(b)
Table 6 Comparison of circle shaped DGS based on the number of columns

\begin{tabular}{lllll}
\hline Design & $\boldsymbol{S}_{11}(\mathrm{~dB})$ & $\boldsymbol{f}_{\boldsymbol{r}}(\mathrm{GHz})$ & $\boldsymbol{S}_{21}(\mathrm{~dB})$ & $\begin{array}{l}\text { Impedance } \\
\text { bandwidth }\end{array}$ \\
\hline Single column circle shaped DGS & -30.13 & 8.34 & -5.28 & $0.72 \mathrm{GHz}$ \\
Two column circle shaped DGS & -30.69 & 8.53 & -1.01 & $1.77 \mathrm{GHz}$ \\
Three column circle shaped DGS & -20.57 & 7.43 & -4.91 & $1.97 \mathrm{GHz}$ \\
\hline
\end{tabular}

Table 7 Dimensions of hexagonal shaped DGS

\begin{tabular}{ll}
\hline Parameters & Units $(\mathrm{mm})$ \\
\hline$H_{l}$ & 2 \\
$H_{b}$ & 1.5 \\
$H_{g}$ & 1 \\
$d$ & 1.4 \\
\hline
\end{tabular}

value because the VNA used was limited to $8 \mathrm{GHz}$ of the bandwidth. Therefore, the bandwidth value is calculated up-to $8 \mathrm{GHz}$.

The findings of the experimentations with the proposed modifications of CPW transmission line DGS affect have applications in the area of embedded antenna system.

The embedded microwave system uses microwave circuits and filters for en-routing the microwave signals from one part of the circuit to another. The microwave filters are specially designed components which are placed between the signal generator and respective antenna operating at particular frequency. Therefore, filter is used to pass certain frequency with a specific bandwidth to the antenna that it is operating at the same frequency. The filter makes the system bulky due to its insertion.

The modified CPW transmission line with DGS is an onboard filter which is placed between the signal generator and antenna. The transmission line itself selects an appropriate frequency and the bandwidth required for the corresponding antenna. There is no additional circuit requirement for filtering of the signals. As this study provides all the frequencies around $5 \mathrm{GHz}$ to $8 \mathrm{GHz}$, this can be used for Wi-Max applications where the service 


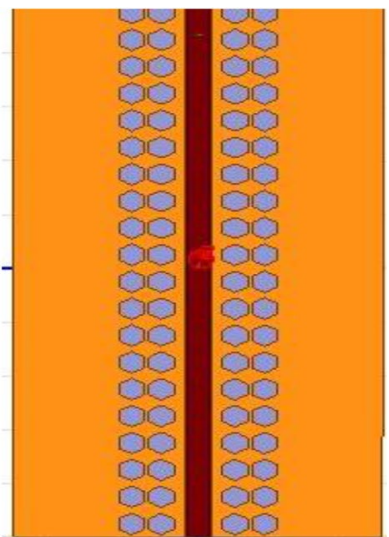

(a)

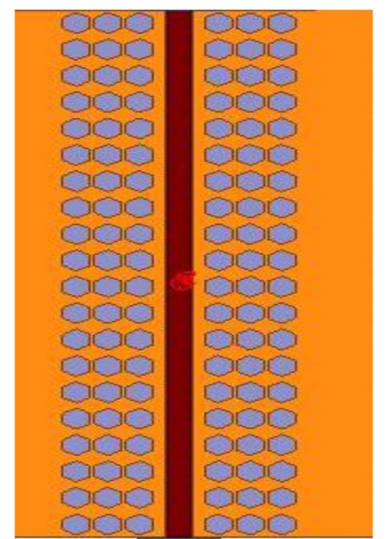

(b)

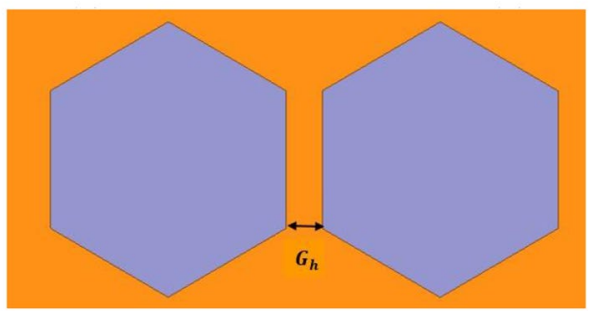

(c)

Fig. 13 a CPW line with two columns of hexagonal shaped DGS b CPW line with three columns of hexagonal shaped DGS c Horizontal distance between the two columns

provider distributes the internet connections using channels to the customers depending on speed of the internet based on the bandwidth of the channel required by a customer.

\section{Discussion and conclusion}

The present paper is related to the study of a CPW transmission line with DGS having periodic unit cells along its both sides. The unit cells are de-metalized from a coplanar ground surface. The selection of the shapes of the slotted unit cells was carried out with a lot of simulations as well as the dimensions of the shape were optimized for $50 \Omega$ characteristic impedance of the CPW line. The number of unit cells also varies according to their shapes for a better impedance match.

From the results, it is predominantly noted that the naturally occurring circular shape offers better results for $S_{11}$ and $S_{21}$. Additionally, the hexagonal shape of a unit cell gives a transmission bandwidth up to $2 \mathrm{GHz}$. However, there is always a trade off between the selection of parameters and its performance.

The transmission loss is also a prime factor for the selection of a unit cell shape and therefore, a circular shaped unit cell based design was fabricated and tested. There is a good agreement between the simulated and fabricated results. All the CPW designs presented in this paper are suitable for Wi-MAX applications where a wide bandwidth is needed. Depending on the frequency requirement, a particular shaped unit cell can be used slotted in ground plane to move the signal along the PCB in high speed microwave channels. Further, the shapes of unit cells can be modified for obtaining ISM band applications of CPW line. Further, the linked ground surface can be incorporated to reduce its transmission loss.
Fig. 14 a $S_{11}$ and $S_{21}$ graph of CPW line with two columns of hexagonal shaped DGS b $\boldsymbol{S}_{11}$ and $\boldsymbol{S}_{21}$ graph of three columns of hexagonal shaped DGS

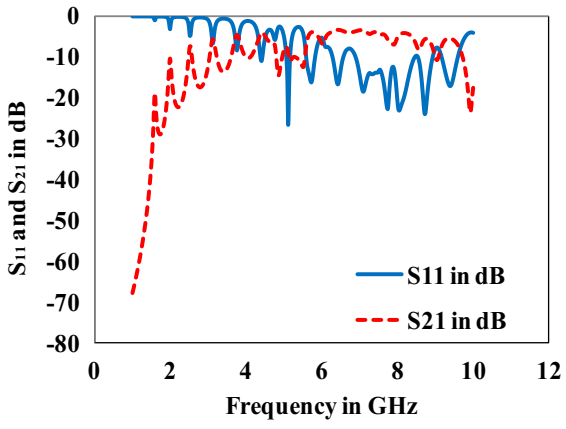

(a)

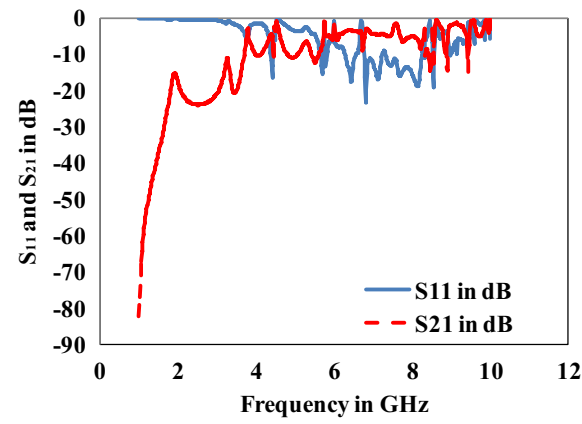

(b)
Table 8 Comparison of the hexagonal shaped DGS based on the number of columns

\begin{tabular}{lllll}
\hline Design & $\boldsymbol{S}_{11}(\mathrm{~dB})$ & $\boldsymbol{f}_{\boldsymbol{r}}(\mathrm{GHz})$ & $\boldsymbol{S}_{21}(\mathrm{~dB})$ & $\begin{array}{c}\text { Impedance } \\
\text { bandwidth }\end{array}$ \\
\hline Single column hexagon shaped DGS & -27.88 & 6.48 & -3.37 & $0.46 \mathrm{GHz}$ \\
Two column hexagon shaped DGS & -23.96 & 8.71 & -5.68 & $2.08 \mathrm{GHz}$ \\
Three column hexagon shaped DGS & -23.11 & 6.80 & -3.35 & $2.03 \mathrm{GHz}$ \\
\hline
\end{tabular}


Fig. 15 a Fabricated CPW line with two column circle shaped DGS b Simulated and measured reflection coefficient for 2 column circle shaped DGS

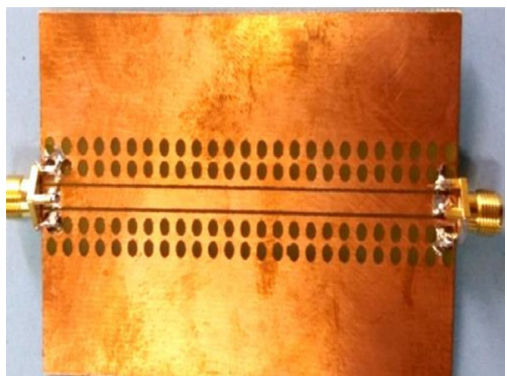

(a)

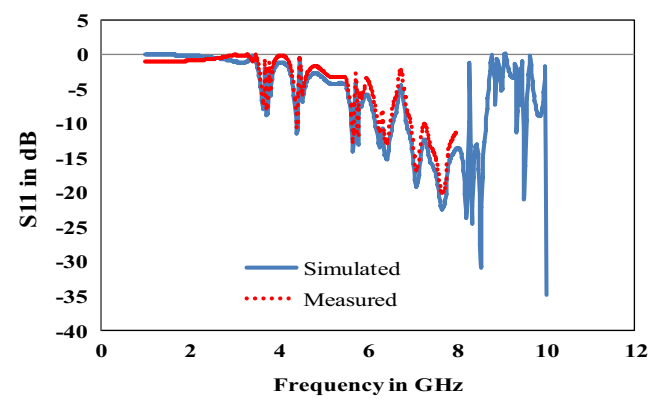

(b)
Table 9 Comparison between simulated and measured results

\begin{tabular}{lll}
\hline Parameter & Simulated & Measured \\
\hline Return loss $S_{11}(\mathrm{~dB})$ & -31.18 & -30.06 \\
Resonant frequency $f_{r}(\mathrm{GHz})$ & $7.65 \mathrm{GHz}$ & $7.81 \mathrm{GHz}$ \\
Impedance Bandwidth & $1.43 \mathrm{GHz}$ & $1.10(+) \mathrm{GHz}$ \\
\hline
\end{tabular}

\section{Compliance with ethical standards}

Conflict of interest The authors declare that they have no conflict of interest.

\section{References}

1. Boutejdar A, Nadim G, Amari S, Omar AS (2005) Control of bandstop response of cascaded microstrip low-pass-bandstop filters using arrowhead slots in backside metallic ground plane. IEEE Antennas and Propag Soc Int Symp 1B:574-577

2. Balalem A, Ali AR, Machac J, Omar A (2007) Quasi-elliptic microstrip low-pass filters using an inter-digital DGS slot. IEEE Microw Wirel Compon Lett 17(8):586-588

3. Liu HW, Li ZF, Sun XW (2003) A novel fractal defected ground structure and its application to the low-pass filter. Microw Opt Technol Lett 39(6):453-456

4. Lu H, Furuya R, Sawyer BMD, Nair C, Liu F (2016) Design, modeling, fabrication and characterization of $2-5 \mu \mathrm{m}$ redistribution layer traces by advanced semi-additive processes on low-cost panel-based glass interposers. IEEE Trans Compon Packag Manuf Technol 6(6):959-967

5. Rezaee M, Joodaki M (2016) Two-dimensional displacement sensor based on CPW line loaded by defected ground structure (DGS) with two separated transmission zeroes. IEEE Sens J

6. Hou Z-Z (2008) Novel wideband filter with a transmission zero based on split-ring resonator DGS. Microw Opt Technol Lett 50(6):1691-1693

7. El-Shaarawy HB, Coccetti F, Plana R, El-Said M, Hashish EA (2010) Novel reconfigurable defected ground structure resonator on co-planar waveguide. IEEE Trans Antennas Propag 58(11):3622-3628
8. Sharma A, Khanna P, Shinghal K, Kumar A (2016) Design of CPWfed antenna with defected substrate for wideband applications. Int J Antenna Propag, 2017

9. Lim JS, Kim CS, Lee YT, Ahn D, Nam S (2002) A spiral-shaped defected ground structure for co-planar waveguide. IEEE Microw Wirel Compon Lett 12(9):330-332

10. Her ML, Wang YZ, Chang CM, Lin KY (2004) Co-planar waveguide (CPW) defected ground structure (DGS) for band-pass filter application. Microw Opt Technol Lette 42(4):331-334

11. Weng LH, Guo YC, Shi XW, Chen XQ (2008) An overview on defected ground structure. Prog Electromagn Res B 7:173-189

12. Hamad EK, Safwat AM, Omar AS (2005) 2D periodic defected ground structure for co-planar waveguide. In: German microwave conference (GeMiC), Germany

13. Mahmud MZ, Islam MT, Misran N, Kibria S, Samsuzzaman M (2018) Microwave imaging for breast tumor detection using uniplanar AMC based CPW-fed microstrip antenna. IEEE Access 6:44763-44775

14. Kumar A,Das S, Yadava RL (2018) DGS and SRR Based FSS Microstrip Antenna for $5 \mathrm{G}$ communication. In: International conference on "computational intelligence and communication technology"

15. Jilani SF, Alomainy A (2018) Millimetre-wave T-shaped MIMO antenna with defected ground structures for $5 \mathrm{G}$ cellular networks. IET Microw Antennas 12(5):672-677

16. Wadell BC (1991) Transmission line design handbook. ISBN 0-89006-436-9, Artech House

17. Ferrari P, Vilcot A (2011) Tunable bandstop defected ground structure resonator using reconfigurable dumbbell-shaped co-planar waveguide. IEEE Trans Microw Theory Tech 54(9):3559-3564

18. Al-Nuaimi MK (2011) Compact CPW-fed antenna loaded with dumbbell slot defected ground structure. In: Antennas and Propagation Conference, Loughborough, UK. pp 14-15

19. Das At, Menon Dsk (2015) Analysis of co-planar waveguide and asymmetric co-planar waveguide fed DGS band-stop filter. Int J Appl Eng Res, 10(55)

20. Khandelwal MK, Kanuajia BK (2017) Defected ground structure: fundamentals, analysis, and applications in modern wireless trends. Int J Antennas Propag, 2017

Publisher's Note Springer Nature remains neutral with regard to jurisdictional claims in published maps and institutional affiliations. 